\title{
Intercambios sobre la filmografía de Thomas Lilti, su incidencia en la formación de médicos cubanos
}

\author{
Daniel Sebastián GARCÍA TORRES
}

Facultad de Medicina No. 1 Universidad de Ciencias Médicas de Santiago de Cuba (Cuba).

Autor para correspondencia: Daniel García Torres. Correo electrónico: danielgarcia@infomed.sld.cu

Recibido el 11 de julio de 2018; aceptado el 13 de septiembre de 2018.

Cómo citar este artículo: García Torres DS. Intercambios sobre la filmografía de Thomas Lilti, su incidencia en la formación de médicos cubanos. Rev Med Cine [Internet] 2019;15(2): 89-93.

DOI: http://dx.doi.org/10.14201/rmc20191528993

\section{Resumen}

El adiestramiento a médicos empleando la filmografía de Thomas Lilti se erige en un ente facilitador al profesor y al educando de un profundo análisis de la práctica profesional, cuestionar la pertinencia de su encargo y de las instituciones hospitalarias en las que la sociedad deposita la salud individual y colectiva de la población, en este sentido sobresalen filmes como Hipócrates y Un doctor en la campiña exponentes de las situaciones complejas de la vida y de las relaciones humanas.

Palabras clave: Hipócrates, salud, estudiantes de medicina, residentes de medicina, médicos rurales.

\section{Exchanges on the filmography de Thomas Lilti, their incidence in the formation of cuban doctors}

\section{Summary}

The training to doctors using Thomas' filmography Lilti is erected in an entity facilitator to the professor and to the educating of a deep analysis of the professional practice, to question the relevancy of their responsibility and of the hospital institutions in those that the society deposits the population's individual and collective health, in this sense films like Hippocrate and The Country Doctor exponents of the complex situations of the life and of the human relationships.

Keywords: Hippocrate, Health, Medicine Students, Medicine Residents, Rural Doctors. 


\section{Introducción}

Una profesión muy importante y de gran trascendencia social: El médico. Los deslices suelen ser fatales al comprometer la existencia física. En los conocimientos y enseñanza del profesor de Medicina está la base de ese profesional que puede, llegado el momento, intervenir a su propia familia. De ahí la importancia que se le concede a la formación ética de estos entes formadores que asientan su conceptualización en el juramento hipocrático.

Un realizador francés y sus dos filmes sobre este tema se ha granjeado la crítica internacional: Thomas Lilti y sus audiovisuales Hipócrates / Hippocrate (2014) y Un doctor en la campiña / The Country Doctor (2016).

En Hipócrates Thomas Lilti sustenta su argumentación en el conocido compromiso de los galenos abordando con certeza principios y contradicciones a las que se enfrentan sus personajes principales.

En su última producción, Un doctor en la campiña, el clínico y cineasta dirige su atención a los médicos rurales, su jerarquía en pequeñas comunidades y a otro elemento de gran interés que es el propio especialista en la condición de enfermo y por lo tanto de paciente.

\section{Fichas técnicas}

Título: Hipócrates.

Titulo original: Hippocrate.

País: Francia.

Año: 2014.

Director: Thomas Lilti.

Música: Sylvain Dewynter, Alexandre Lier, Nicolas Weil.

Fotografía: Nicolas Guarin

Montaje: Christel Dewynter

Guion: Thomas Lilti, Baya Kasmi, Pierre Chosson, Julien Lilti

Interpretes: Vincent Lacoste, Jacques Gamblin, Reda Kateb, Marianne Dennicourt, Félix Moati, Carole Franck, Philippe Rebbot, Julie Brochen, Jeanne Cellard, Thierry Levaret...

Duración: 102 minutos.

Género: comedia, drama, medicina.

Idioma original: francés.

Color: color.

Productora: 31 Juin Fims, Agnés Vallée, Emamnuel Barraux

Resumen: Benjamín está destinado a ser un gran doctor, pero su primera experiencia como médico residente en el hospital donde trabaja su padre, no sale como él esperaba. La práctica se revela mucho más compleja que la teoría, y la responsabilidad es aplastante. Además, su compañero de trabajo, un médico extranjero, tiene mucha más experiencia que él. Benjamín tendrá que enfrentarse cara a cara con sus límites y sus miedos, así como los de sus pacientes y sus familiares, los médicos y sus compañeros residentes.

\section{Enlaces:}

https://www.imdb.com/title/tt2891070

https://www.filmaffinity.com/es/film890404.html

\section{$\underline{\text { Trailer }}$}

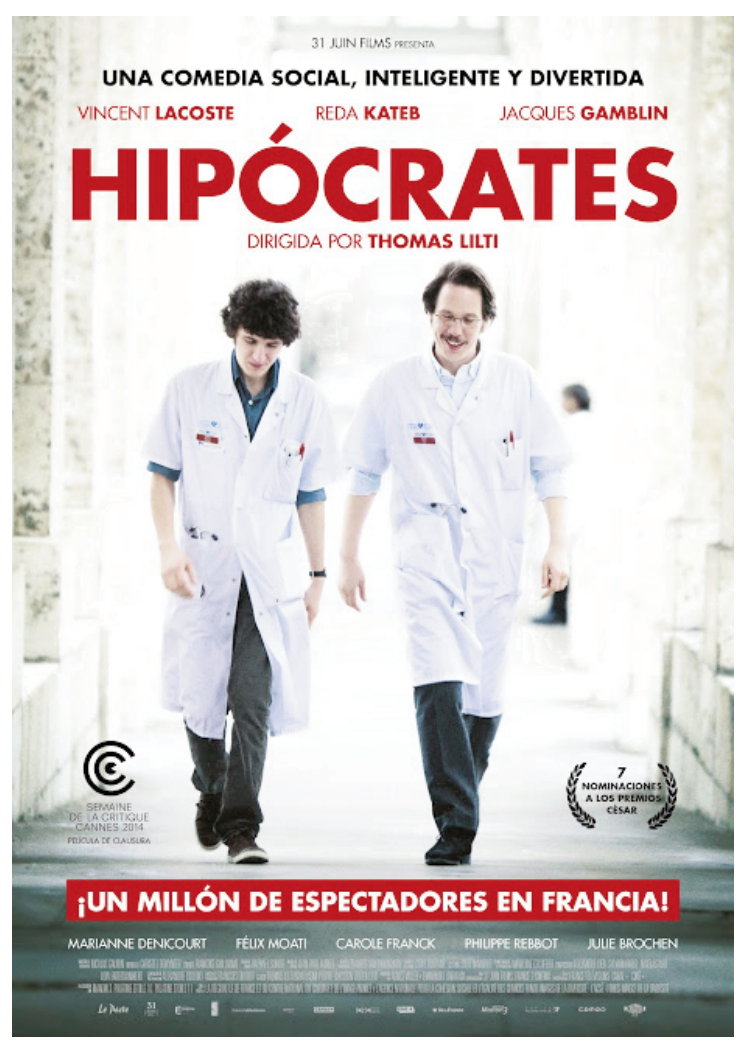

Título: Un doctor en la campiña.

Título original: Médecin de champagne.

País: Francia.

Año: 2016.

Director: Thomas Lilt

Musica: Alexandre Lier, Sylvain Ohrel, Nicolas Weil.

Fotografía:Nicolas Gaurin. 
Montaje: Christel Dewynter.

Guion: Thomas Lilti, Baya Kasmi.

Interpretes: François Cluzet, Marianne Denicourt, Isabelle Sadoyan, Félix Moati, Patrick Descamps

Duración: 102 minutos.

Género: ficción.

Idioma original: francés.

Color: color.

Resumen: "Todas las personas que viven en esta zona rural pueden contar con Jean-Pierre, el médico que les ausculta, cura y tranquiliza a cualquier hora del día, los siete días de la semana. Enfermo a su vez, acepta la llegada de Nathalie, recién llegada de su trabajo de hospital, para que le ayude" (cineuropa).

Productoras: 31 Juin Films, France 2 Cinéma, Le Pacte.

Enlaces:

https://www.imdb.com/title/tt5078326

https://www.filmaffinity.com/es/film646143.html

Trailer

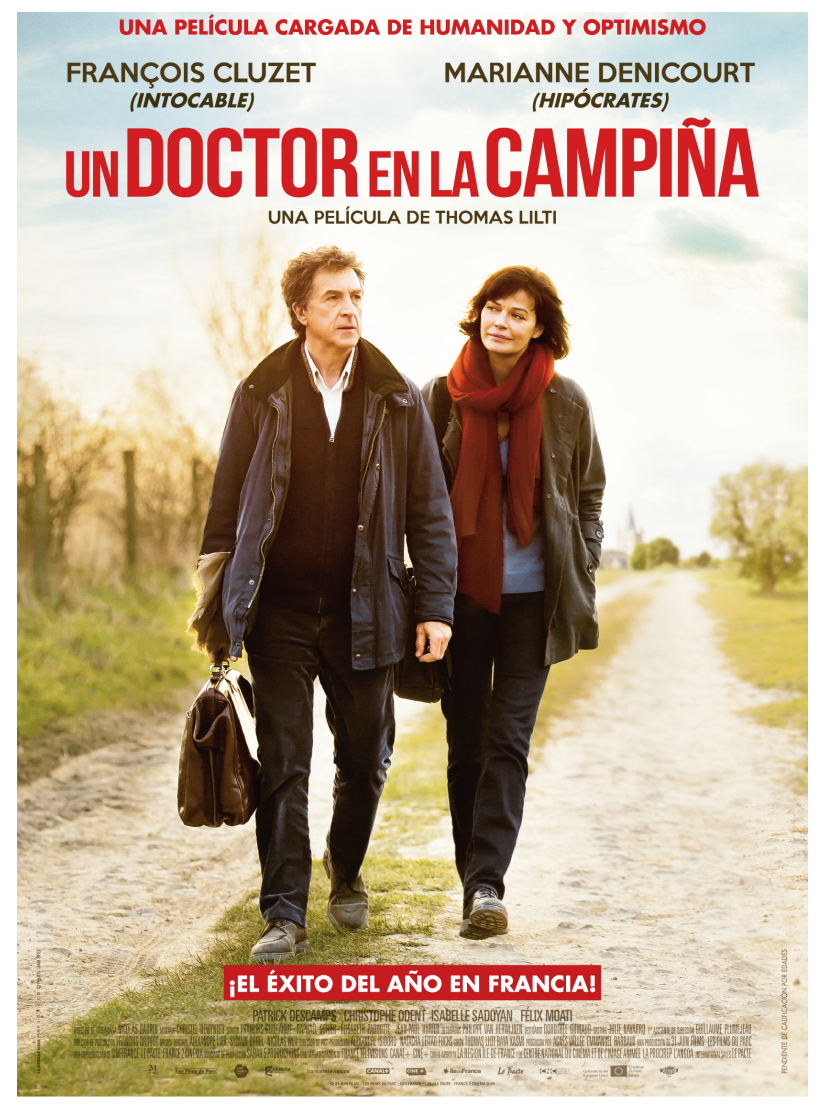

\section{Debates e incidencia en la formación educativa}

En trabajos anteriores se ha argumentado acerca de las excelentes oportunidades de los audiovisuales para incidir en la formación de los estudiantes de Medicina a la vez que se cultiva la inteligencia y el conocimiento que propicia el séptimo arte ${ }^{1,2}$.

La creación de espacios para el debate cinematográfico con este tipo de discípulos, resulta oportuno para favorecer una cultura profesional, siempre y cuando sea sostenido en un periodo de tiempo y compartido con la participación de especialistas en cada materia.

Materiales sobre Medicina abundan e incluso los de corte comercial se distribuyen ampliamente a nivel global. Series como Emergencias / Emergency Room (1994) la mejor de todas; Anatomía de Grey / Grey's anatomy (2005); El doctor House / House M. D. (2004); Chicago Med (2015); Esperanza / Saving Hope (2012); Código Negro / Black Code (2015); The Knick (2015) sorprende por una trama rica en valores y agudeza critica a la época; El buen doctor / The Good Doctor (2017) han recreado a millones de espectadores. En muchas de ellas médicos con poderes extravasados del mítico Olimpo o autistas casi androides resuelven disimiles casos, otros más humanos enfrentan al telespectador con las dificultades de la praxis médica. Todo lo contrario, a la serie argentina Nueve Lunas / Nine Moons (1994) conducida por Fernando Bassi e interpretada por Oscar Martínez y Cecilia Roth en la que resalta el amor y el humanismo hacia la vida, la profesión y sobre todo hacia las mujeres.

En Hipócrates su realizador y miembro de esta humana profesión, camino a una autoexpresión clínica, confronta al público con una realidad aguda, la verdadera vida de un hospital, las relaciones interpersonales que de ella se derivan a la cual se adecuan los estudiantes de Medicina en Cuba en un proceso práctico instructivo que comienza desde los primeros años de la carrera.

Thomas Lilti asevera refiriéndose a su experiencia en hospitales que están lastrados por problemas como el nepotismo y el corporativismo, y por el exceso de burocracia y jerarquía 3 .

En un artículo publicado en la Revista de Medicina y Cine sus autores valoran que esta película puede ser una excelente herramienta pedagógica mostrando la verdadera cara del servicio asistencial, así como en el adiestramiento bioeticista del médico. Aseveran que el director de forma sutil e irónica aprovecha diferentes momentos de la película para criticar la 
situación de la sanidad pública francesa: falta de material, falta de personal sanitario, bajos salarios y visión económica de los gestores ${ }^{4}$. Mal arraigado como cáncer incurable que lacera y corrompe y no entiende de andamiaje de sistema político.

El médico lleva a cabo su profesión mediante un compromiso con la ciencia médica y con los enfermos, a base de profesionalismo cuyo concepto clave es la colocación de los intereses del paciente por encima de los del médico, obligando al doctor a mantener estándares de competencia profesional y constituirse en el auténtico experto para la sociedad en el terreno de la salud 5 .

En Hipócrates se evidencia que a pesar de la importancia del médico en el servicio se ejecutan procesos de ajustes de plantilla o recortes de sanidad con las consecuentes nefastas consecuencias. En países más pobres suelen ser devastadores pues se les problematiza aún más el acceso a los servicios hospitalarios $6,7,8$.

Contrastando con la realidad cubana que protege al profesional de la salud y le sitúa en el lugar primordial que le corresponde como salvaguarda de la integridad de la salud individual y colectiva a pesar de las carencias en centros hospitalarios muy comunes pues debe sobreponerse al impacto del bloqueo económico más terrible, despiadado e inmoral sufrido en los últimos decenios $9,10,11$.

A pesar de ello se garantiza el adiestramiento de miles de doctores y residentes incluso de otras naciones. En el filme un residente extranjero, en este caso argelino, Abdel, sufre la discriminación al foráneo en tierra ajena y debe esforzarse más para conquistar sus metas. La educación de los extranjeros desde la óptica cubana es modelo del optimismo y del desinterés pues los recibe en sus instituciones con un carácter distinguido por la comunidad profesional. Cuba los acoge y amamanta con la sabia de la experiencia como a sus hijos. El profesor cubano sabe que su aporte se revierte en la población de disímiles latitudes y continentes ${ }^{12}$.

No podemos soslayar que en Hipócrates se introduce el controvertido tema de las relaciones entre las generaciones de médicos, abordado críticamente un aspecto medular para el médico consagrado que busca facilitar el camino de un hijo, en ocasiones a costa de lacerar los intereses de éste. El hijo de un médico puede encontrar simpatías en quienes idolatran al padre, o superponerse con lo opuesto si el discípulo no es de los más aventajados, en este caso el venerado progenitor recibirá de algunos la crítica mordaz o la ayuda desleal.
También aborda la negligencia en el servicio, denuncia las componendas poco éticas de quienes con errores ponen en peligro o causan la muerte a los pacientes a su cuidado, la gestión privada de los servicios públicos, afirma que en Francia tienen el mismo problema que en España en cuanto a la sanidad. Los hospitales públicos son gestionados por entes privados a los cuales sólo les interesa la rentabilidad y no la salud de los pacientes ${ }^{13}$.

En Un doctor en la campiña, Lilti muestra un ambiente más pausado propio de los pueblos en el campo, matizado por el sabor de lo cotidiano, de costumbres repetidas, pero sano, se vive sin agitaciones.

En los campos cubanos la panorámica humanística de Lilti es similar a la que muestra en su filme. Los servidores de la salud encuentran un mundo rico en tradiciones populares, en la benevolencia del campesino que pasa su tiempo labrando la tierra o dedicado a otras tareas algunas importadas de las ciudades.

En Cuba los consultorios médicos están diseminados por todas partes llevando la solidaridad y la cultura preventiva a todos los rincones del país. El joven graduado es acogido con amor y hacen gala de su profesión dedicándose por completo a los pacientes y las dolencias típicas que se presentan.

Los graduados realizan sus primeros años como profesionales, allí en plena campiña, el médico de la familia es el guía en la educación de la comunidad en materia de salud, promoviendo la prevención de enfermedades, es el primero que llega ante un caso grave, accidente o lesiones no intencionales. En lugares recónditos y de difícil acceso, se le advierte caminando o montando a caballo hasta la casa del campesino. Antes del triunfo de la revolución había pocos médicos que llevaban a cabo esta inmensa labor y que incluso ante la precaria situación ni cobraban los servicios que prestaban al labriego empobrecido14.

Lilti propicia polemizar estos temas de salud pública a partir de la muestra de la situación en Francia, expresado mediante una vía que usualmente no es seleccionada para ello: el cine. Su filmografía aborda la realidad, sin tapujos ni enmiendas. En una recrea la formación de los residentes en la otra el médico experto. Dos facetas opuestas propuestas a estudiantes y residentes para analizar, debatir aprender. Razones muy apreciadas en la preparación de los futuros médicos que provee con matices nuevos la formación cultural complementaria del buen arte y la pertinencia de la universidad hacia la profesión. 


\section{Referencias}

1. García Torres DS, Sánchez Hechavarría M, Montoya Ledel M, Franco Díaz C, Díaz Suárez R. El Cine Club como vía para el debate y tratamiento a la educación preventiva antidrogas en estudiantes de medicina. Rev Med Cine 2016; 12(4):236-9.

2. García Torres DS, Fuentes Domínguez N, Mendoza Ruiz M, Díaz Suárez R, Perera Díaz J. El cine debate con estudiantes de medicina para incidir preventivamente sobre el sexo transaccional. Rev Med Cine 2018; 14(4): 245-251.

3. Salva N. Thomas Lilti: El sistema médico es defectuoso y absurdo. El Periódico. [Internet]. 8 de junio de 2015 [citado 15 Jun 2018].

4. Pintor Holguin E, Herreros Ruiz Valdepenas B, Gargantilla-Madera P, Vivas-Rojo E. Hipócrates (2014). La realidad de un residente en un hospital público. Rev Med Cine 2017; 13(4): 171-6.

5. Núñez Corté JM. Valores del médico y su carácter. FEM 2014; 17(supl 1):S23S37.

6. Vicente B de. La crisis internacional y la austeridad golpean a los niños en países de altos ingresos. UNICEF Comité Español [Internet]. 13 de abril de 2017 [citado 15 Jun 2018].

7. Un estudio internacional alerta del impacto en la salud de los recortes. La Voz de Galicia [Internet]. 5 de marzo de 2013 [citado 22 Jun 2018].

8. Andreu Segura B. Recortes, austeridad y salud. Informe SESPAS 2014. Gac Sanit. 2014;28(S1):7-11.

9. Marimón Torres N, Torres Martínez Er. Efectos del bloqueo económico, financiero y comercial de Estados Unidos en el Sistema Nacional de Salud. Rev Cubana Salud Pública 2013; 39 (2): 298-313.

10. Rosales Reyes SÁ, García Triana BE, Raimundo Padrón E, Valverde Grandal O. Repercusión del bloqueo económico, comercial y financiero de Estados Unidos en la educación médica superior cubano. Rev Haban Cienc Méd [Internet]. 2013, 12(suppl5):92-101.

11. Noticia. Daños del bloqueo en la salud superan los 87 millones de dólares. CUBADEBATE. [Internet] 12 Oct 2017 [citado 23 Jun 2018].

12. Peña Pentón D, Con tanta ciencia como conciencia. Impacto de la Escuela Latinoamericana de Medicina en el contexto internacional actual. Panorama Cuba y Salud [Internet]. 2015; 10 (2): 38-42.

13. Dorado M. Hipócrates: La denuncia social hecha comedia. Zilencine [Internet] 7 de mayo 2015 [citado 23 Jun 2018].

14. García Torres DS. Dr. Hugo Orlando Torres Aldrich: Ejemplo de profesional en la praxis de la pediatría cubana. MEDISAN [Internet]. 2016; 20(2): 267-76. 